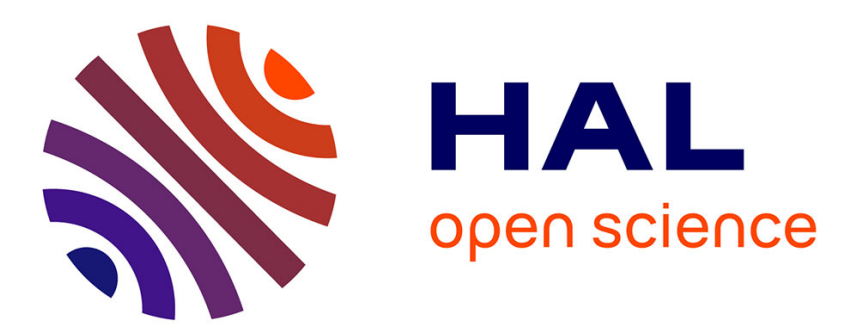

\title{
Between additive and subtractive color mixings: intermediate mixing models
}

\author{
Lionel Simonot, Mathieu Hébert
}

\section{To cite this version:}

Lionel Simonot, Mathieu Hébert. Between additive and subtractive color mixings: intermediate mixing models. Journal of the Optical Society of America. A Optics, Image Science, and Vision, 2013, 31 (1), pp.58-66. 10.1364/JOSAA.31.000058 . hal-00962256

\section{HAL Id: hal-00962256 https://hal.science/hal-00962256}

Submitted on 20 Sep 2014

HAL is a multi-disciplinary open access archive for the deposit and dissemination of scientific research documents, whether they are published or not. The documents may come from teaching and research institutions in France or abroad, or from public or private research centers.
L'archive ouverte pluridisciplinaire HAL, est destinée au dépôt et à la diffusion de documents scientifiques de niveau recherche, publiés ou non, émanant des établissements d'enseignement et de recherche français ou étrangers, des laboratoires publics ou privés. 


\title{
Between additive and subtractive color mixings: intermediate mixing models
}

\author{
Lionel Simonot ${ }^{1 * *}$ and Mathieu Hébert ${ }^{2}$ \\ ${ }^{1}$ Institut Pprime, Université de Poitiers, CNRS UPR 3346, Département Physique et mécanique \\ des matériaux, SP2MI, BP 30179, 86962 Chasseneuil, Futuroscope Cedex, France \\ ${ }^{2}$ Université de Lyon, Université Jean Monnet de Saint-Etienne, CNRS UMR5516, \\ Laboratoire Hubert Curien, F-42000 Saint-Etienne, France \\ ${ }^{*}$ Corresponding author: lionel.simonot@univ-poitiers.fr
}

Received July 18, 2013; revised October 16, 2013; accepted November 11, 2013; posted November 13, 2013 (Doc. ID 194145); published December 5, 2013

\begin{abstract}
The color rendering of superposed coloring components is often an issue either to predict or to simulate the appearance of colored surfaces. In graphical software, for example, transparence options are available to display different layouts on top of each other. With two colored layers, tuning the transparency of the top layer enables transitioning continuously from the color of this top layer to the color of the bottom layer. However, these options based very often only on additive color mixing offer limited transitions between two base colors. It would be advantageous to introduce more advanced options, providing, for example, realistic renderings of superposed paint layers. This is the aim of the present study, where simple models are proposed to create intermediate configurations between additive and subtractive color mixings. These models rely on the spectral power distribution of a finite set of primaries with given proportions. They may be extended to RGB color reflective or transmissive systems if the red, green, and blue wavebands do not overlap each other. An additional parameter is introduced to tune the proportions of additive and subtractive mixings, each type of mixing being based on its set of primaries. Various simulations of color mixings are presented, illustrating the possibilities offered by this model in addition to those permitted by the purely additive and subtractive mixings. (c) 2013 Optical Society of America

OCIS codes: $\quad$ (300.6550) Spectroscopy, visible; (330.1690) Color; (330.1730) Colorimetry.

http://dx.doi.org/10.1364/JOSAA.31.000058
\end{abstract}

\section{INTRODUCTION}

What is the result of the mixing between different colors? The answer of course depends on the relative proportions of the different primaries, but also on the type of color mixing. In additive mixing, color is obtained by mixing lights with different spectral power distributions either by projection of colored light beams or by juxtaposition of small colored areas as in displays. The colors are synthesized by mixing at least three primaries, generally not more: red, green, and blue. In subtractive mixing, colors are obtained by superposing colored layers behaving as spectral filters for white light, like the inks in half-tone printing. Three primaries are commonly used: cyan, magenta, and yellow. The distinction between additive and subtractive color mixing was first exposed by Helmholtz [1] in 1860 in order to clarify the previous confusion between light and colorant mixture. In many color ordering systems [2] and in the habits of painters even today, the primaries are often red, yellow, and blue. There is a large dispersion in the precise definition of these primaries depending on the available pigments. In practice, e.g., for painting, the mixing laws are complex to simulate. Nevertheless, the resulting color in general stays between those predicted by the ideal additive and by the ideal subtractive mixing. In half-tone printing, Yule and Nielsen [3] defined such an intermediate empirical mixing law. In this paper, we propose other easily understandable color mixing laws governed by intuitive parameters: the component proportions and a mixing parameter indicating whether the color synthesis is additive or subtractive. The ideal additive and subtractive mixing are included in these new mixing laws as limit cases. The mixing laws deal with spectral data with a minimum of three spectral bands. The models can also be satisfyingly extended to RGB color systems in the case in which the wavebands defining the red, green, and blue channels do not overlap each other.

\section{DEFINITION OF COLORS}

The color $\mathbf{P}$ of a surface comes from the interpretation by the human visual system of the light signal reflected or transmitted by the surface, especially its spectral distribution denoted $P_{\lambda}$. The number $N$ of wavebands depends on the measurement system. At least three wavebands in the visible range are needed to define colors, but $N$ may be larger, or much larger in the cases of multi and hyper-spectral systems. By normalizing the measured spectrum by a reference spectrum considered as the maximum signal (direct incident light for transmittance measurements, and light scattered by a white standard for reflectance measurements), the spectrum satisfies the property

$$
0 \leq P_{\lambda} \leq 1 \quad \forall \lambda
$$

The color $\mathbf{P}$ can be considered as a vector in an $N$ dimensional space (with $N \geq 3$ ), and $P_{\lambda}$ are its coordinates. From the spectral values, the color can be defined more conventionally by using the standardization of the CIE $[4,5]$. The CIEXYZ tristimulus values are obtained by multiplying the 
spectral value $P_{\lambda}$, the standardized illuminant radiance spectrum $S_{\lambda}$, and each of the matching color functions $\bar{x}_{\lambda}, \bar{y}_{\lambda}$, and $\bar{z}_{\lambda}$, and then summing the resulting spectrum over the visible wavelength domain:

$$
\left\{\begin{array}{l}
X=k \sum_{\lambda} P_{\lambda} S_{\lambda} \bar{x}_{\lambda} \\
Y=k \sum_{\lambda} P_{\lambda} S_{\lambda} \bar{y}_{\lambda}, \\
Z=k \sum_{\lambda} P_{\lambda} S_{\lambda} \bar{z}_{\lambda}
\end{array}\right.
$$

where the factor $k$ is a normalization factor giving $Y=100$ for the reference whose spectral values are $P_{\lambda}=1$ for every waveband. For object colors, the CIELAB color space is classically used. The coordinates-lightness $L^{*}$, green/red coordinate $a^{*}$, and blue/yellow coordinate $b^{*}$ — can be derived from the CIEXYZ tristimulus values by nonlinear relations $[4,5]$.

\section{COLOR MIXING RULES}

Let us consider $N_{c}$ primaries $\mathbf{P}_{i}$ with $i \in\left\{1, \ldots, N_{c}\right\} . N_{c}$ is commonly 2 (binary mixing) or 3 (ternary mixing). But $N_{c}$ can be larger to precisely model color reproduction systems; for example, for RGB displays, the black background can be numbered among the primaries $\left(N_{c}=4\right)$, or in a CMY trichromy, the bare paper, or the paper with one, two, or three ink layers, are all considered as primaries $\left(N_{c}=8\right)$. Several color mixing configurations can be defined from the spectra $\mathbf{P}_{i, \lambda}$. The following rules are an attempt to formalize some of these configurations.

Let us denote as $\mathbf{P}$ the color obtained by mixing the $N_{c}$ primaries $\mathbf{P}_{i}$ with respective proportions $c_{i}$ satisfying the conditions

$$
0 \leq c_{i} \leq 1
$$

and

$$
\sum_{i=1}^{N} c_{i}=1 .
$$

- Rule 1: The spectral values $P_{\lambda}$ attached to $\mathbf{P}$ satisfy the property (1).

- Rule 2: The proportions $c_{i}$ should have the same influence on $P_{\lambda}$ for every waveband $\lambda$ in the visible spectrum.

- Rule 3: The mixing operation is commutative: $\mathbf{P}$ is independent of the ordering of the components in the mixing.

- Rule 4: By considering the mixing between two colors with respective proportions $c$ and $1-c$, the relation $P_{\lambda}(c)$ is continuous and monotonous with $c$ in every waveband $\lambda$.

Rule 4 induces the following logical property: the color resulting from the mixing of color $\mathbf{P}_{1}$ with itself is again color $\mathbf{P}_{1}$ for every proportion $c$.

It is important to note that counterexamples can be found where these rules are not satisfied, e.g., color mixing with photoluminescence effects where light emission at one wavelength greatly depends on the absorption at a smaller wavelength.

\section{A. Purely Additive and Subtractive Mixing Laws}

The color mixing laws may be illustrated by considering a composite filter, or "color cell," of unit area and unit thickness

decomposed into different subareas and/or different sublayers as shown in Fig. 1. The following assumptions are made regarding the optical properties of subareas and sublayers:

- They do not scatter light and therefore satisfy BeerLambert-Bouguer law (i.e., the transmittance of superposed components is the product of the component's individual transmittances).

- The multiple reflections of light between superposed components are not taken into account.

- Lateral propagation of light between neighboring subareas can be neglected.

These strong assumptions allow defining some ideal mixing laws respecting the previously stated rules.

Let us denote as $a_{i}$ the fractional areas of the subareas, and as $t_{i}$ the fractional thickness of the sublayers.

In purely additive mixing, the color cell is composed of $N_{c}$ juxtaposed filters of unit thickness that thus represent the primaries $\mathbf{P}_{i}$ [Fig. 1(a) for $N_{c}=3$ ]. The fractional areas of these subareas, $a_{i}$, satisfying

$$
\sum_{i=1}^{N_{c}} a_{i}=1,
$$

are the proportions $c_{i}$ of the mixing. The additive mixing is a linear combination of the primary spectral values:

$$
P_{\lambda}=\sum_{i=1}^{N_{c}} c_{i} P_{i, \lambda}
$$

with $c_{i}=a_{i}$.

\section{(a) Purely additive mixing}
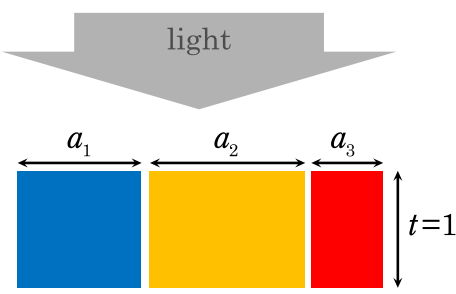

(b) Purely subtractive mixing

(c) Yule-Nielsen mixing

(d) Additive -subtractive mixing
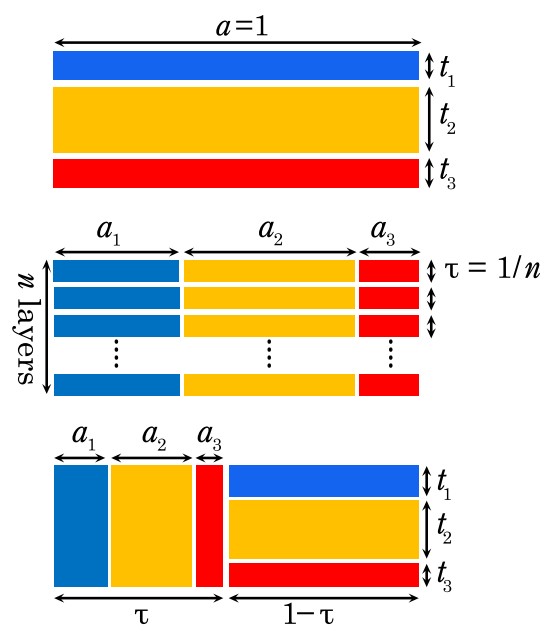

(e) Subtractive -additive mixing

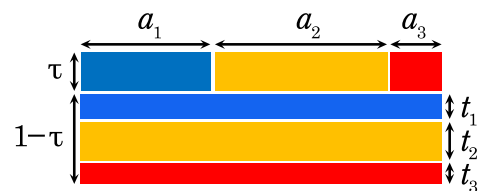

Fig. 1. Color cells describing the different mixing laws. 
In purely subtractive mixing [see Fig. $\underline{1(\mathrm{~b})}$ for $N_{c}=3$ ], the color cell is defined as the superposition of $N_{c}$ sublayers of unit area with respective fractional thicknesses $t_{i}$, satisfying

$$
\sum_{i=1}^{N_{c}} t_{i}=1
$$

These fractional thicknesses are the proportions $c_{i}$ of the mixing. The purely subtractive mixing consists in a weighted geometric mean of the color components:

$$
P_{\lambda}=\prod_{i=1}^{N_{c}} P_{i, \lambda}^{c_{i}}
$$

with $c_{i}=t_{i}$.

Both the additive and the subtractive mixing laws verify the mixing rules, except the continuity (Rule 4) in the case of the subtractive mixing when one spectral value $P_{j, \lambda}$ of at least one primary is zero. In this case, $P_{\lambda}=0$ for every $0<c_{j} \leq 1$ and $P_{\lambda}=\prod_{i \neq j} P_{i, \lambda}^{c_{i}}$ for $c_{j}=0$. This discontinuity may yield an aberrant color for the mixing. That is the reason why we restrict the mixing rules to strictly positive values. Condition (1) thus becomes

$$
0<P_{\lambda} \leq 1 \quad \forall \lambda .
$$

In practice, zero values are replaced with the minimal measurable value.

\section{B. Kubelka-Munk Paint Mixing Model}

Purely additive and subtractive color mixing are ideal cases. The color rendering of painting mixings is more complex to predict. The Kubelka-Munk model [6] is a widely used approach to simulate the reflectance spectra of paintings as a function of the absorption coefficient $K$ and the backscattering coefficient $S$ of the paint. For an opaque layer, the reflectance $R$ is directly related to the ratio $K / S$ :

$$
\frac{K}{S}=\frac{(1-R)^{2}}{2 R}
$$

When $N_{c}$ different paints are mixed, one generally assumes the additivity of the absorption and backscattering coefficients $K_{i}$ and $S_{i}$ of the paints [묘, $\underline{]}$, i.e.,

$$
\frac{K}{S}=\frac{\sum_{i}^{N_{c}} c_{i} K_{i}}{\sum_{i}^{N_{c}} c_{i} S_{i}},
$$

where $c_{i}$ represent the concentrations of the respective components.

The reflectance of an infinite layer of this paint can then be calculated by inverting the relationship (10), but both $K_{i}$ and $S_{i}$ for every component must be known. These optical parameters are rather difficult to determine because at least two spectral measurements are required, e.g., the reflectance of the sample layer on a black and on a white substrate. A database of absorption and scattering coefficients of pigments can then be created in the visible range [9] or in a larger range 350-2500 $\mathrm{nm}[10,11]$. This technique is efficient only if exactly the same pigments with the same binding as in the database are used. Unfortunately, the $K_{i}$ and $S_{i}$ values are largely dependent on the morphology and on the environment of the pigments, which are generally not known with precision. Therefore, it is not possible to predict painting color mixing correctly by using only the spectral data $P_{i, \lambda}$, considered in this case as the spectral reflectance of the component $i$ in an opaque form.

\section{Kubelka's Layering Model}

An alternative model for "intensively light-scattering materials" was proposed by Kubelka in 1954 [12]. It relies on the composition of layers characterized by their spectral reflectance $R_{i, \lambda}$ and transmittance $T_{i, \lambda}$. By taking into account the multiple reflections between the layers, the reflectance of the specimen viewed at the side of layer 1 is

$$
R_{\lambda}=R_{1, \lambda}+\frac{T_{1, \lambda}^{2} R_{2, \lambda}}{1-R_{1, \lambda} R_{2, \lambda}},
$$

and the transmittance (viewed at either side) is

$$
T_{\lambda}=\frac{T_{1, \lambda} T_{2, \lambda}}{1-R_{1, \lambda} R_{2, \lambda}} .
$$

However, this model does not satisfy the commutativity law (Rule 3 stated above): the color in reflection mode depends on the ordering of the layers. If the individual reflectances of the layers are sufficiently small to be ignored, we have a superposition of nonscattering layers and, as expected, Eq. (13) becomes equivalent to the multiplicative color mixing, and, by introducing the fractional thicknesses $t_{i}$, to the purely subtractive mixing law [Eq. (요)].

To overcome the limitation imposed by the purely additive or purely subtractive laws, more empirical mixing laws described by a color cell are presented hereinafter with the restricting assumption of no light scattering within the colored components.

\section{Half-tone Printing Color Mixing}

Purely additive or subtractive mixing laws are also incapable of predicting half-tone colors produced by printing. According to the Murray-Davies model [13], the half-tone color is a linear combination of the spectral reflectances of the different inked areas as in purely additive mixing [Eq. (6)]. The model is generalized to the case of three inks by the Neugebauer model [14], where the primaries are the paper background, the paper areas covered by each ink, and the paper areas covered by two or three inks. Since this model takes explicitly into account neither the lateral propagation of light within the paper bulk nor the internal reflections at the paper-air interface, its predictions are not accurate. To overcome this drawback, Yule and Nielsen [3] introduced a corrective equation where the individual primary reflectances are raised to the power $1 / n$ and the sum is raised to the power $n$, with $n$ a parameter to be fitted:

$$
P_{\lambda}=\left(\sum_{i=1}^{N_{c}} c_{i} P_{i, \lambda}^{1 / n}\right)^{n},
$$

where $N_{c}$ is the number of colorants including those obtained by the superposition of inks and including the bare background ( $N_{c}=2^{k}$ colorants for $k$ inks printing), $c_{i}$ is the fractional area coverage of the corresponding colorant, and $P_{i, \lambda}$ represents the spectral values attached to this colorant (e.g., its spectral reflectance). 
In the original model, three spectral bands were considered. The spectral version of this equation based on thinner wavebands, also known as the Yule-Nielsen modified spectral Neugebauer model, was later proposed by Viggiano [15].

It is worth noting that the additive mixing law [Eq. (ㅁ)] (i.e., the classical Neugebauer model) is a special case of Eq. (14) with $n=1$. Moreover it can be shown [16] that the subtractive mixing law [Eq. (8)] can be defined as the limit case of Eq. (14) when $n$ tends to $+\infty$. In other words, the empirical parameter $n$ enables intermediate mixing laws from the additive mixing for $n=1$ to the subtractive mixing for $n \rightarrow+\infty$. By setting $\tau=1 / n$, Eq. (14) becomes

$$
P_{\lambda}=\left(\sum_{i=1}^{N_{c}} c_{i} P_{i, \lambda}^{\tau}\right)^{1 / \tau}
$$

The color cell representing the Yule-Nielsen model is shown in Fig. 1(c): it is the superposition of $n$ identical sublayers of thickness $\tau$, composed of $N_{c}$ subareas of fractional area $a_{i}$. The mixing proportions are $c_{i}=a_{i}$, and the new parameter $\tau=1 / n$ indicates if the mixing is subtractive $(\tau \approx 0)$ or additive $(\tau \approx 1)$. Note that after each sublayer, the lights attenuated by the different subareas are mixed before reaching the next sublayer.

This model is a first example of color cell containing both sublayers and subareas, a concept that is extended in the next section.

\section{NEW COLOR MIXING LAWS}

As an alternative to the Yule-Nielsen model, we suggest other intermediate mixing laws between purely additive and purely subtractive mixings by defining color cells as presented in Figs. $\underline{1(\mathrm{~d})}$ and $\underline{1(\mathrm{e})}$ for $N_{c}=3$. The color cells are the junction of two cells, one subdivided into subareas with fractional areas $a_{i}$ (additive mixing) and one subdivided into sublayers with fractional thickness $t_{i}$ (subtractive mixing). The relative proportions of these two cells are parameterized by a mixing parameter $\tau$ varying between 0 for the ideal subtractive mixing [Eq. (8)] and 1 for the ideal additive mixing [Eq. (ㅁ)]. For both cells, the proportions $c_{i}$ of each primary are respected. This principle yields two configurations, which we call additivesubtractive mixing and subtractive-additive mixing.

\section{A. Additive-Subtractive Mixing}

The color cell contains $N_{c}$ subareas with unit thickness (additive mixing) and one additional subarea made of $N_{c}$ sublayers (subtractive mixing). From Fig. 1(d), the mixing parameter $\tau$ is defined as

$$
\tau=\sum_{i=1}^{N_{c}} a_{i}
$$

while

$$
\sum_{i=1}^{N_{c}} t_{i}=1
$$

The proportions $c_{i}$ attached to the different primaries can also be calculated from the fractional areas $\alpha_{i}$ and fractional thicknesses $t_{i}$ using the following formula:

$$
c_{i}=a_{i}+(1-\tau) t_{i}
$$

In order to ensure the same proportion of each component in both the additive and the subtractive parts, we set

$$
\left\{\begin{array}{l}
a_{i}=\tau c_{i} \\
t_{i}=c_{i}
\end{array}\right.
$$

The additive-subtractive law can be expressed either in terms of the color cell parameters, i.e., the fractional areas $a_{i}$ and thicknesses $t_{i}$,

$$
P_{\lambda}=\sum_{i=1}^{N_{c}} a_{i} P_{i, \lambda}+\left(1-\sum_{i=1}^{N_{c}} a_{i}\right) \prod_{i=1}^{N_{c}} P_{i, \lambda}^{t_{i}},
$$

or in terms of the intuitive parameters, i.e., the proportions of primaries $c_{i}$ and the mixing parameter $\tau$ :

$$
P_{\lambda}=\tau \sum_{i=1}^{N_{c}} c_{i} P_{i, \lambda}+(1-\tau) \prod_{i=1}^{N_{c}} P_{i, \lambda}^{c_{i}} .
$$

\section{B. Subtractive-Additive Mixing}

The color cell is divided into $N_{c}$ sublayers with unit area (subtractive mixing) and one additional sublayer of thickness $\tau$ made of $N_{c}$ subareas (additive mixing). We see from Fig. 1(e) that the mixing parameter $\tau$ is defined as

$$
\tau=1-\sum_{i=1}^{N_{c}} t_{i}
$$

while

$$
\sum_{i=1}^{N_{c}} a_{i}=1
$$

The proportions $c_{i}$ of primaries can be also calculated from the fractional areas $a_{i}$ and the fractional thicknesses $t_{i}$ :

$$
c_{i}=t_{i}+\tau a_{i} .
$$

We set

$$
\left\{\begin{array}{l}
a_{i}=c_{i} \\
t_{i}=(1-\tau) c_{i}
\end{array}\right.
$$

As for the additive-subtractive mixing, the subtractiveadditive law can be expressed either in terms of the color cell parameters, i.e., the fractional areas $a_{i}$ and thicknesses $t_{i}$,

$$
\left.P_{\lambda}=\left(\sum_{i=1}^{N_{c}} a_{i} P_{i, \lambda}^{\left(1-\sum_{i=1}^{N_{c}} t_{i}\right.}\right)\right) \cdot \prod_{i=1}^{N_{c}} P_{i, \lambda}^{t_{i}},
$$

or in terms of the intuitive parameters, i.e., the proportions of primaries $c_{i}$ and the mixing parameter $\tau$ :

$$
P_{\lambda}=\left(\sum_{i=1}^{N_{c}} c_{i} P_{i, \lambda}^{\tau}\right) \cdot \prod_{i=1}^{N_{c}} P_{i, \lambda}^{c_{i}(1-\tau)} .
$$

\section{Examples of Spectral and Colorimetric Variations}

Figure 2 shows the spectra resulting in mixing between blue and yellow by additive mixing [Fig. 2(a) and Eq. (6)], 
subtractive mixing [Fig. 2(b) and Eq. (ㅁ)], additive-subtractive mixing [Fig. 2(c) and Eq. (21) with $\tau=0.5$ ], and subtractiveadditive mixing [Fig. 2(d) and Eq. (27) with $\tau=0.5$ ].

All figures clearly show the monotonous variations with the concentration. As the blue and yellow spectra have the same value at $505 \mathrm{~nm}$, this value is constant and independent of the proportions of primaries for this wavelength.

Figure 3 shows the corresponding colorimetric variations between yellow and blue but also between blue and red and between red and yellow in the CIELab space. The calculations are performed from the simulated spectra and by using the standard illuminant D65 and the standard observer defined by the CIE $\left(1931,2^{\circ}\right)$. The subtractive mixing always offers darker colors than the additive mixing [Fig. 3(a)]. In the chromatic plane [Fig. 3(b)], the differences may be important (yellow and blue, or red and blue mixing) or weak (yellow and red mixing). The colorimetric variations for the mixing laws defined in Eqs. (21) and (27) are intermediates between ideal additive and subtractive mixing. For the same mixing parameter $\tau=0.5$, the additive-subtractive law is rather close to the additive law while the subtractive-additive law is rather close to the subtractive law.

\section{Comparison between Mixing Laws}

The additive-subtractive mixing law defined by Eq. (21), the subtractive-additive mixing law defined by Eq. (27), and the Yule-Nielsen modified spectral Neugebauer mixing law defined by Eq. (15) are three different spectral and colorimetric ways to progressively go from the ideal subtractive mixing to the ideal additive mixing. In order to compare these laws, we consider binary mixings of equal proportion $(c=0.5)$ between the primaries blue and yellow whose spectral variations are presented in Fig. 2. Color gradients are computed according to each mixing law by varying $\tau$ from 0 to 1 . For comparison, the mixing parameter $\tau$ is $1 / n$ in the case of the Yule-Nielsen model. The color deviation from the additive-subtractive or the subtractive-additive mixing law to the Yule-Nielsen modified

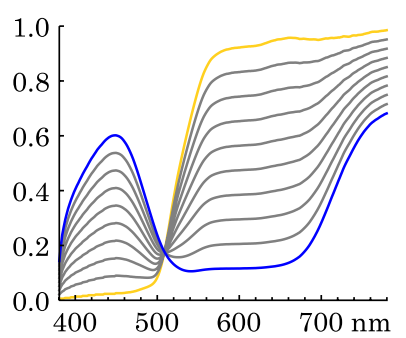

(a)

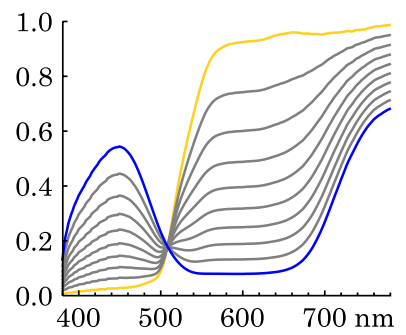

(c)

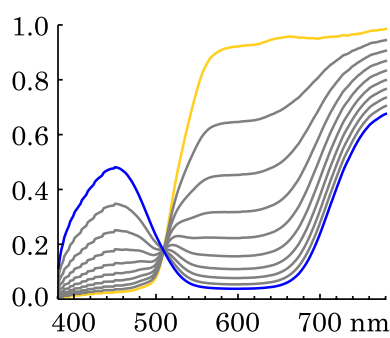

(b)

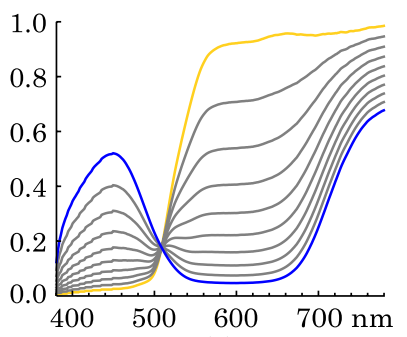

(d)
Fig. 2. Spectra resulting in binary color mixing $\left(N_{c}=2\right)$ between blue and yellow. (a) Additive mixing, (b) subtractive mixing, (c) additive-subtractive mixing (with $\tau=0.5$ ), and (d) subtractiveadditive mixing (with $\tau=0.5$ ). The proportion varies from 0 to 1 by steps of 0.1 .

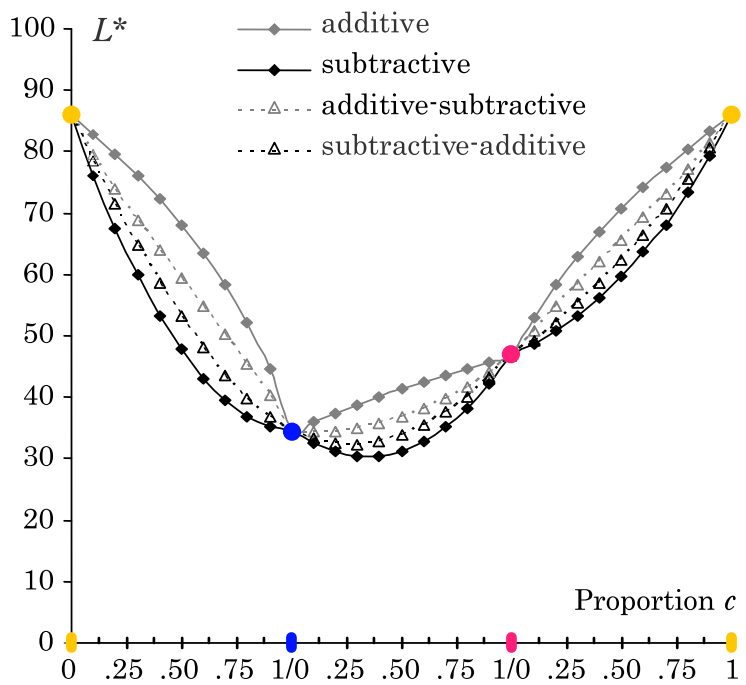

(a)

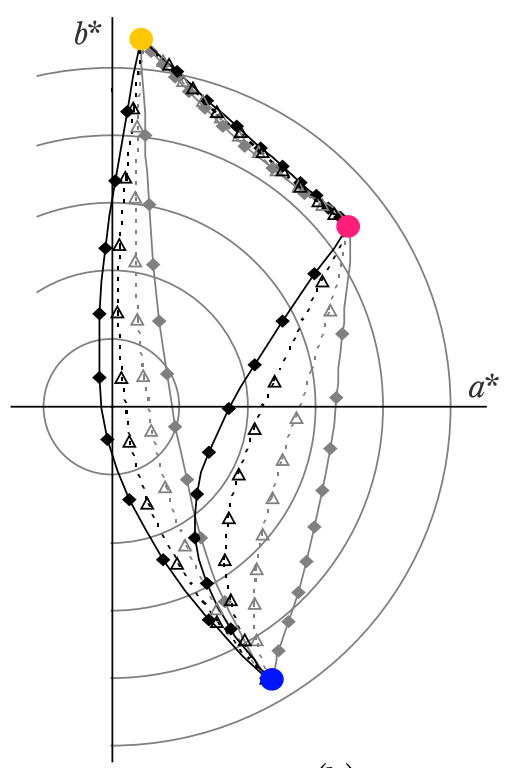

(b)

Fig. 3. CIELab coordinates resulting from different binary color mixings between yellow, blue, and red. (a) Variation of the lightness $L^{*}$ in terms of the proportion and (b) variation in the $\left(a^{*}, b^{*}\right)$ plane. The proportion varies from 0 to 1 by steps of 0.1 .

spectral Neugebauer mixing law is expressed in terms of CIELAB 1976 color distance $\Delta E$ and plotted as a function of $\tau$ in Fig. 4 . The three mixings are obviously equivalent when $\tau=0$ and $\tau=1$, and therefore $\Delta E=0$. The color differences between the Yule-Nielsen and the additive-subtractive mixing are weak and would not be perceptible by a human observer $(\Delta E<1)$. Let us note the very particular case for a binary color mixing with $c=0.5$ and $\tau=0.5$ where both mixing laws give the same results $(\Delta E=0)$. The differences between the Yule-Nielsen modified spectral Neugebauer and the subtractive-additive mixing laws are more significant, reaching even larger values when $\tau=0.5$ ( $\Delta E=8.9$ units).

\section{EXTENSION TO RGB COLOR MIXING}

In computing devices, the classical 24-bit colors are stored as three integers between 0 and 255 . In most software, only 


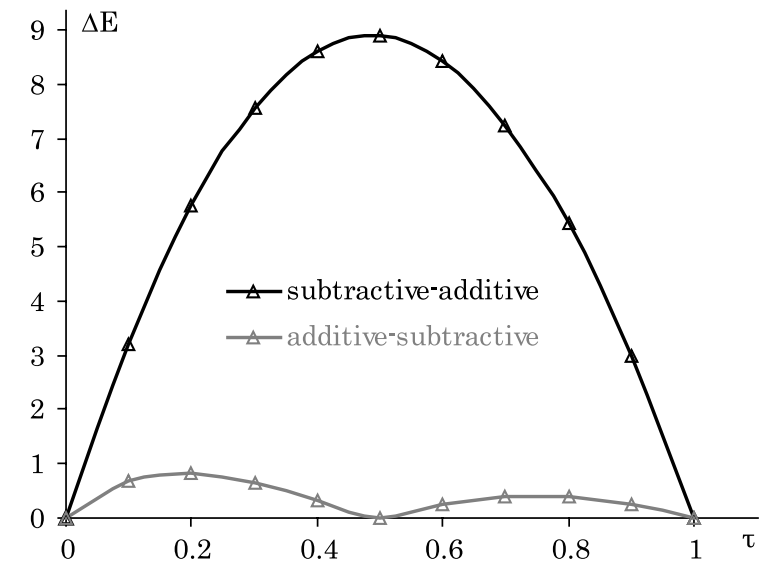

Fig. 4. Variation as a function of the mixing parameter $\tau$ of the color difference $\Delta E$ between the Yule-Nielsen modified spectral Neugebauer mixing laws and the additive-subtractive (gray line) or subtractive-additive (black line) mixing law, for a binary color mixing with $c=0.5$ between yellow and blue primaries.

additive color mixing is allowed. As the spectral relation (6) is linear, a similar linear relation is established with CIEXYZ tristimulus values [Eq. (2)] and with all other tristimulus values linearly connected to CIEXYZ space, e.g., an RGB space. Therefore, the additive mixing between two RGB colors can be written as

$$
X=\sum_{i=1}^{N_{c}} c_{i} X_{i} \quad \text { with } X=\mathrm{R}, \mathrm{G} \quad \text { or } \quad \mathrm{B} .
$$

Unfortunately, such a direct relation in an RGB space does not exist when the spectral relation is not linear, which is the case for all other color mixing laws [Eqs. ()), (14), (21), and (27)]. Nevertheless, these mixing laws can be used by considering the RGB not as a color space but as three distinct spectral bands. In other words, the RGB values are used as spectral coordinates $P_{\lambda}$ for three different wavelengths $\lambda$.

Figure $\underline{5}$ gives a flowchart explaining the color mixing calculation in the case of $N_{c}=3$ and the equations to be used with RGB values. To ensure the property given by Eq. (9) and especially to avoid zero values, the reduced coordinates $r, g, b$ are calculated as follows:

$$
x=\frac{X+1}{256},
$$

where $x$ denotes $r, g$, or $b$.

The color mixing laws are then applied with these reduced coordinates. The final R, G, and B values of the obtained color are calculated from the reduced coordinates by inverting Eq. (29) and taking the floor value of $X=\mathrm{R}, \mathrm{G}$, or B.

Figure 6 shows the colors obtained with RGB values in the case of additive-subtractive binary mixing [Eq. (21) with $N_{c}=2$ ] between yellow, blue, and red primaries. The mixing parameter $\tau$ obviously provides an additional degree of freedom to define the color mixing.

In color synthesis, three primaries are generally used and therefore the color set can be represented within a triangle. This representation was first proposed by Mayer in the mideighteenth century as the base of its double tetrahedron color solid [17]. A simplified color representation of the Mayer

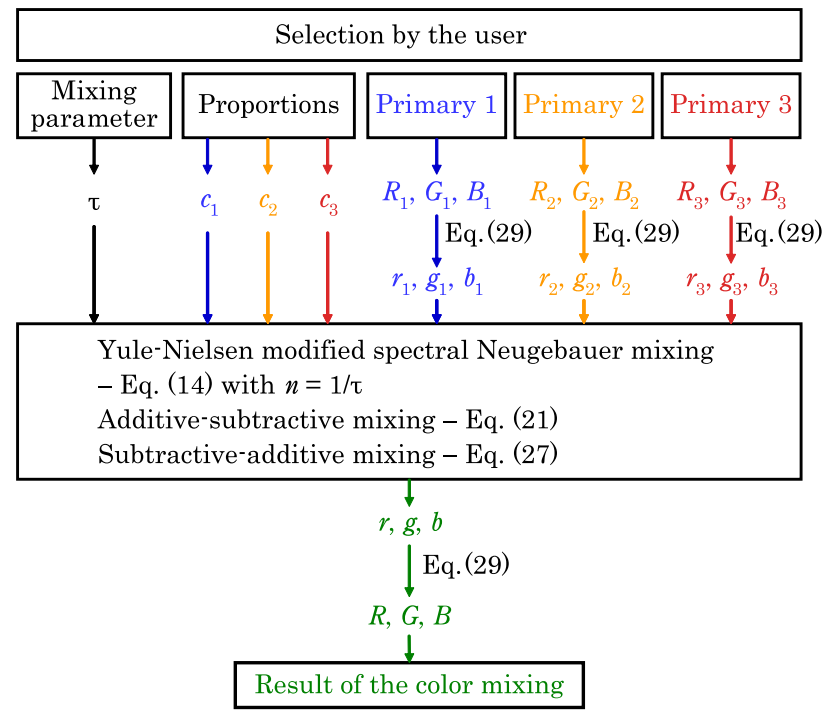

Fig. 5. Flowchart for the color mixing calculation from RGB values with three primaries.

triangle, shown in Fig. 7(a), was given by Lichtenberg in a commentary to the Mayer essay [17] using gamboge, cinnabar, and Prussian blue as primary colorants. Lambert, in collaboration with the painter Benjamin Calau [18], also chose the triangle as the base of its Farbenpyramide and gamboge, cinnabar, and Prussian blue for the triangle vertex. Inspired by Mayer's work, Young [19] illustrates a color mixture diagram in the form of an equilateral triangle based on red, green, and violet as principal colors that he suggested to be directly detected by particles in the retina. Maxwell includes this triangular representation to quantify additive color mixing from red, green, and blue primaries by using either a color top with different painted sections [20] or a light box mixing spectral lights [21]. In modern colorimetry, the color gamut given by additive mixing is located within a triangle in the CIExy chromaticity diagram with red, green, and blue as the most common primaries. But this triangle shape is deformed in the case of subtractive mixing due to the nonlinear mixing law. Figures $7(\mathrm{~b})-7(\mathrm{~d})$ show the colors obtained with RGB values in the case of additivesubtractive ternary mixing [Eq. (21) with $N_{c}=3$ ] between yellow, blue, and red primaries, represented as Lichtenberg [Fig. 7(a)] under the form of a Mayer triangle. The mixing parameter is $\tau=1$ (purely additive mixing) in Fig. $7(\mathrm{~b})$, $\tau=0.5$ in Fig. $7(\mathrm{c})$, and $\tau=0$ (purely subtractive mixing) in Fig. $7(\mathrm{c})$. In the same way, such models could be used to generate color charts for artistic or design applications.

It is worth noting that computing color mixing from the spectral distribution of the primaries and from their RGB color values is not equivalent except in the case of the purely additive mixing. This may be simply shown by mixing a colored light with white light: varying the proportion of each light modifies the lightness and chroma, but the hue should remain constant. However, if processed from the RGB values of the two lights, the color mixing may induce hue shifts. This is illustrated in Fig. 8, where a blue purple $(\mathrm{R}=80, \mathrm{G}=0, \mathrm{~B}=170)$ is mixed with white $(R=255, G=255, B=255)$. Except for the purely additive mixing, the hue shifts to pinkish tint when the white proportion increases. Only purely additive mixing ( $\tau=1$ ) or computation from the spectral values avoid this undesirable hue shift. 


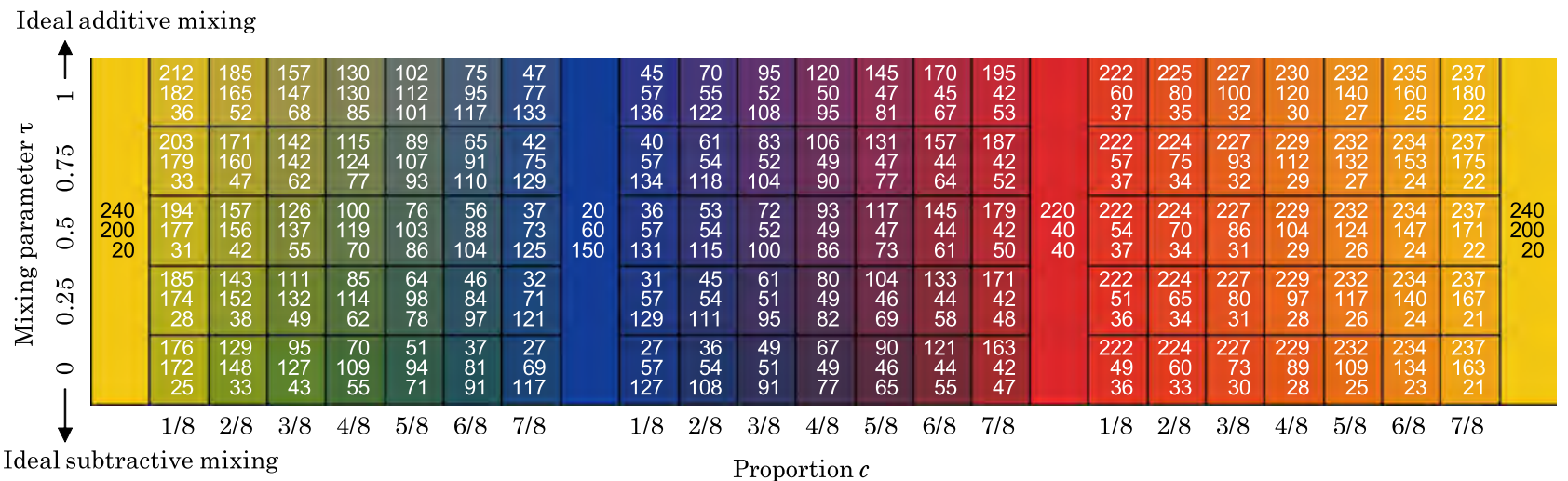

Fig. 6. Binary color mixing in RGB between yellow, blue, and red by using the additive-subtractive mixing law [Eq. (21) with $N_{c}=2$ ]. The proportion $c$ varies from 0 to 1 by steps of 0.1 , and the mixing parameter $\tau$ varies from 0 to 1 by steps of 0.25 . The RGB values are written in each box.

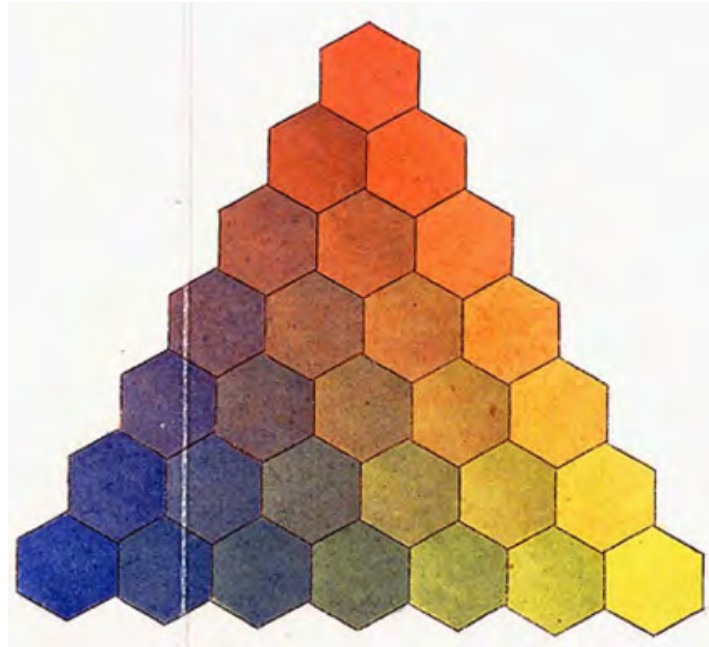

(a)

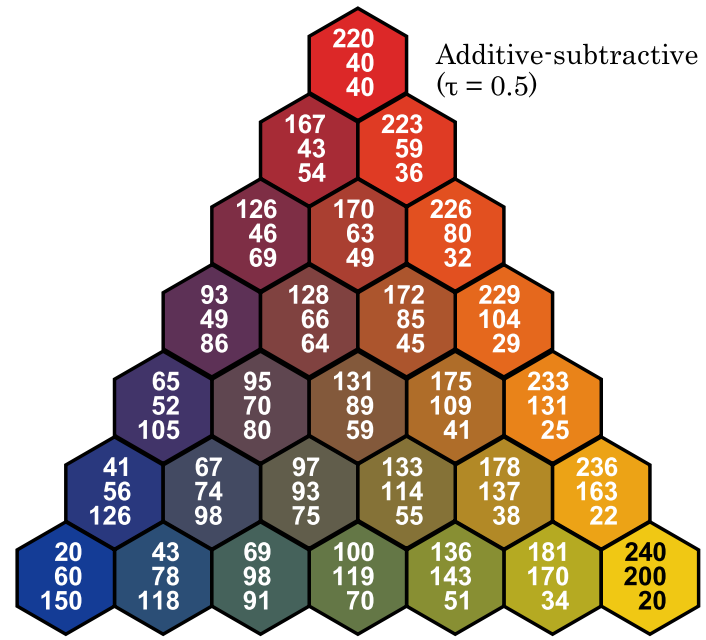

(c)

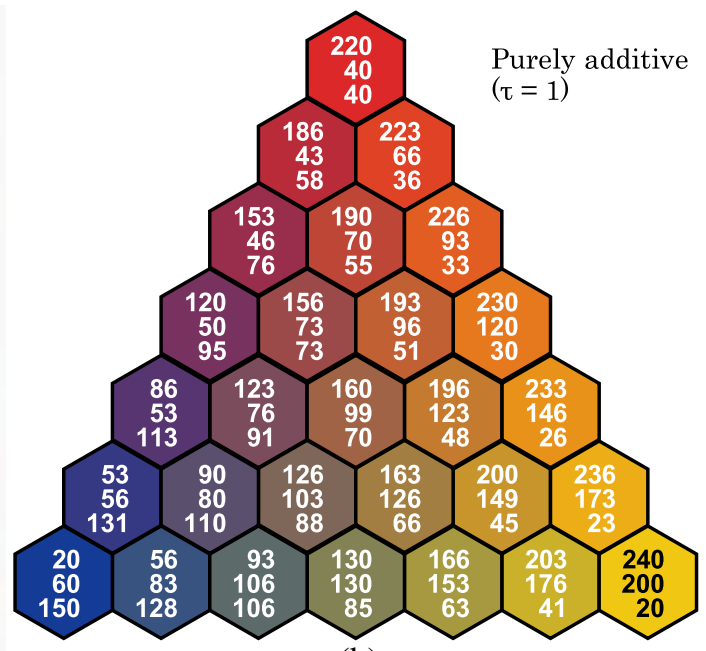

(b)

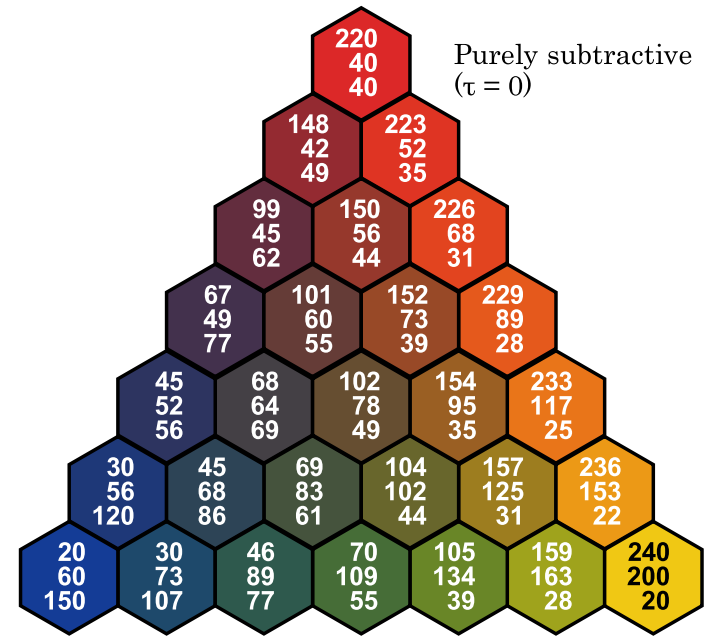

(d)

Fig. 7. Color mixing triangle. (a) Representation of Mayer triangle by Lichtenberg from [16]. (b)-(d) Ternary color mixing in RGB between yellow, blue, and red by using the additive-subtractive mixing law [Eq. (21) with $N_{c}=3$ ] for $\tau=0,0.5$, and 1, respectively. The RGB values are written in each box.

\section{APPLICATION TO TRANSPARENCY}

The transparency illusion, e.g., in an image, is generated under several conditions first studied by Metelli [22]. Among them, the colorimetric changes between the surfaces represented with and without transparency must be coherently interpreted as transparency by the brain [23]. Therefore, the color 


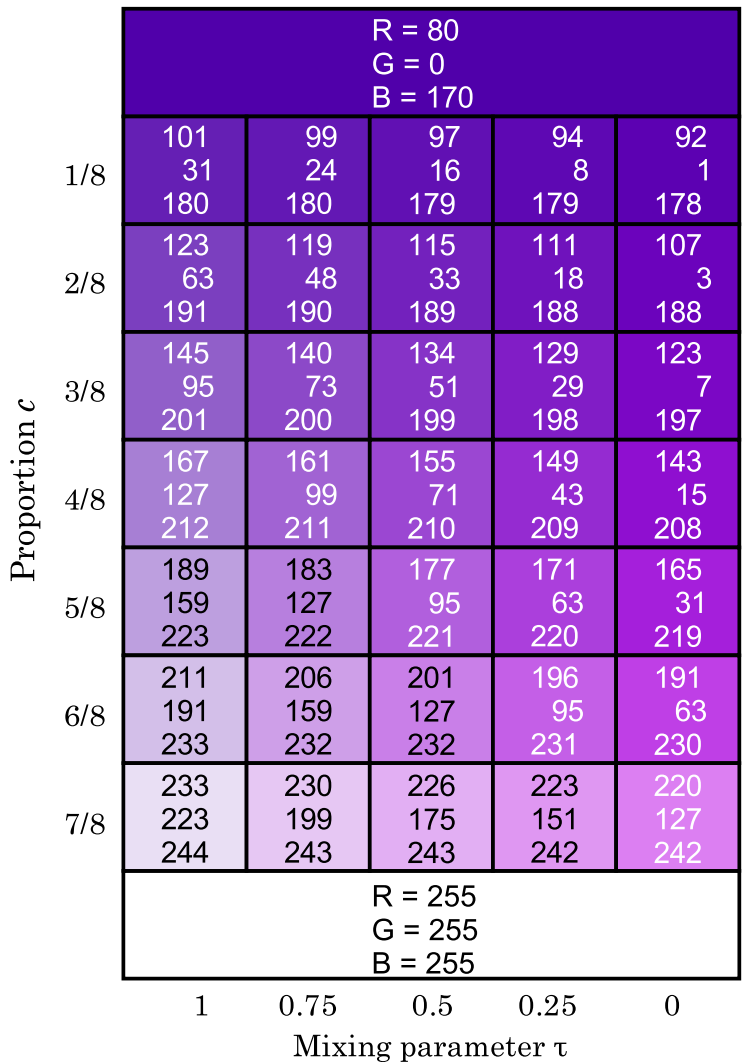

Fig. 8. Binary color mixing computed from the RGB values of blue, purple, and white by using the additive-subtractive mixing law [Eq. (21) with $N_{c}=2$ ]. The proportion $c$ varies from 0 to 1 by steps of 0.1 , and the mixing parameter $\tau$ varies from 0 to 1 by steps of 0.25 . The RGB values are indicated in each color patch.

transparency can be studied as a binary color mixing between a foreground color and a background color. The transparency rate would be equivalent to the proportion $c$ of the background color. An opacity rate can also be defined as $1-c$. The kind of transparency can be chosen by tuning the parameter $\tau$ from the subtractive transparency ( $\tau=0$ to simulate colored optical filters) to the additive transparency $(\tau=1$ to simulate partially pierced surfaces).

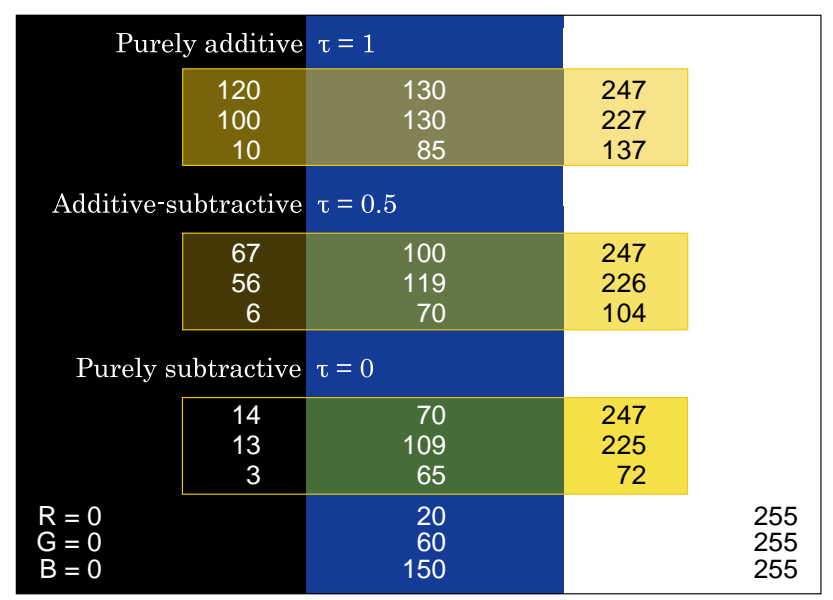

Fig. 9. Color transparency in RGB color system. Foreground color: yellow $(\mathrm{R}=240, \mathrm{G}=200, \mathrm{~B}=20)$ with a transparency rate $c=0.5$. Additive-subtractive mixing [Eq. (26)] for different values of $\tau$. Background colors: black, blue, and white.
Figure $\underline{9}$ shows the result of a yellow transparent color on a black, a blue, and a white background for additive, subtractive, and additive-subtractive (with $\tau=0.5$ ) laws following the RGB calculations presented in Fig. 5. It clearly illustrates the differences between the mixing/transparency laws. Compared to the additive law, the subtractive law gives a very dark color on the black background, a green color on the blue background, and a saturated yellow on the white background. It is the expected color behavior of an absorbing but nonscattering yellow filter. Intermediary cases (e.g., $\tau=0.5$ in Fig. 9 ) allow simulating a filter with some scattering effects. It can particularly be seen on the black background where the yellow color can be made out. This last result shows that even though the individual components of the models are assumed to be nonscattering in order to satisfy the color mixing rules, scattering effects can be simulated.

\section{CONCLUSION}

In this paper, we present easily understood color mixing laws governed by intuitive parameters: the relative proportions of each component and the mixing law parameter. These laws are alternatives of the Yule-Nielsen modified spectral Neugebauer model, specifically used for half-tone printing. This study can be a first step to propose more advanced laws, by taking into account multiple scattering between scattering centers, interface effects, etc. We restrict the study by adopting some color mixing rules, but theses rules can also be extended in order to insert interference effects, fluorescent effects, or goniochromatism.

The mixing laws require acquiring the spectral reflectance and/or transmittance of the different colors. Therefore, the study is well adapted to hyperspectral imaging, where the complete spectrum is collected for each pixel of the image instead of only the three classical RGB values. From a hyperspectral image, color mixing can be applied on the spectral values, and CIE tristimulus values can then be rigorously calculated. However, the mixing laws can also be applied directly to RGB values with in most cases very convenient results, yielding interesting alternatives to the most usual additive mixing law. The mixing parameter allows modifying progressively color mixing from the ideal subtractive to the ideal additive mixing. It can become a very useful tool for software dealing with colors (word, image processing, presentation, drawing software, etc.)

\section{REFERENCES}

1. H. von Helmholtz, Handbuch der Physiologischen Optik (Voss, 1860), Band II, Sektion 20.

2. R. G. Kuehni and A. Schwarz, Color Ordered: A Survey of Color Systems from Antiquity to the Present (Oxford University, 2008).

3. J. Yule and W. Nielsen, "The penetration of light into paper and its effect on halftone reproduction," Proc. TAGA 3, 65-76 (1951).

4. G. Wyszecki and W. S. Stiles, Color Science: Concepts and Methods, Quantitative Data and Formulae, 2nd ed. (Wiley Interscience, 1982).

5. CIE, "Colorimetry," 3rd ed., Technical Report CIE 15 (CIE, 2004).

6. P. Kubelka and F. Munk, "Ein Beitrag zur Optik der Farbanstriche," Zeitschrift für technische Physik 12, 593-601 (1931).

7. D. Duncan, "The colour of pigment mixtures," Journal of Oil Colour Chemistry Association 32, 296-321 (1949).

8. F. Grum and C. J. Bartleson, Optical Radiation Measurement (Academic, 1980), Vol. 2. 
9. G. Latour, M. Elias, and J. M. Frigerio, "Determination of the absorption and scattering coefficients of pigments: application to the identification of the components of pigment mixtures," Appl. Spectrosc. 63, 604-610 (2009).

10. R. Levinson, P. Berdahl, and H. Akbari, "Solar spectral optical properties of pigments-Part I: model for deriving scattering and absorption coefficients from transmittance and reflectance measurements," Sol. Energy Mater. Sol. Cells 89, 319-349 (2005).

11. R. Levinson, P. Berdahl, and H. Akbari, "Solar spectral optical properties of pigments-Part II: survey of common colorants," Sol. Energy Mater. Sol. Cells 89, 351-389 (2005).

12. P. Kubelka, "New contributions to the optics of intensively light scattering material. Part II: non-homogeneous layers," J. Opt. Soc. Am. 44, 330-335 (1954).

13. A. Murray, "Monochrome reproduction in photoengraving," J. Franklin Inst. 221, 721-744 (1936).

14. H. E. J. Neugebauer, "Die Theoretischen grundlagen Des Mehrfarbendruckes," Zeitschrift für wissenschaftliche Photographie, Photophysik und Photochemie 36, 36-73 (1937).

15. J. A. S. Viggiano, "The color of halftone tints," Proc. TAGA 37, $647-661$ (1985)
16. A. Lewandowski, M. Ludl, G. Byrne, and G. Dorffner, "Applying the Yule-Nielsen equation with negative $n$," J. Opt. Soc. Am. A 23, 1827-1834 (2006).

17. T. Mayer, De affinitate colorum commentatio, in Opera inedita Tobiae Mayeri, G. C. Lichtenberg, ed. (Göttingen, 1775).

18. J.H.Lambert, Beschreibung einermitdem Calaunischen Wachse ausgemalten Farbenpyramide, Haude and Spener, eds. (Haude and Spener, 1772).

19. T. Young, A Course of Lectures on Natural Philosophy and the Mechanical Arts, J. Johnson, ed. (Johnson, 1807).

20. J. C. Maxwell, "Experiments on colour, as perceived by the eye, with remarks on colour-blindness," Trans. R. Soc. Edinburgh 21, 275-297 (1855)

21. J. C. Maxwell, "On the theory of compound colours, and the relations of the colours of the spectrum," Philos. Trans. R. Soc. London 150, 57-84 (1860).

22. F. Metelli, "The perception of transparency," Sci. Am. 230, 90-98 (1974).

23. M. D'Zmura, P. Colantoni, K. Knoblauch, and B. Laget, "Color transparency," Perception 26, 471-492 (1997). 